\title{
IX.
}

Aus der medicinischen Klinik des Herrn Prof. Quincke in Kiel.

\section{Ueber Erkrankung der Medulla oblongata im Kindesalter.}

\author{
Von \\ G. Hoppe-Seyler, \\ Privatdocent und Assistenzarzt.
}

In der medicinischen Klinik zu Kiel kam ein Fall von Erkrankung der Medulla oblongata zui Beobachtung, welche aus dem Kindesalter stammte und mit keinem der wenigen Fälle von Bulbäraffectionen im jugendlichen Alter, die bisher beschrieben wurden, vollständig ủbereinstimmt.

- Da nur wenige Angaben über solche Erscheinungen im Kindesalter vorliegen, ist es wohl von Interesse, diesen Fall kurz zu schildern.

Elise B o ck, Arbeiterstochter, 14 Jahre alt, wurde am 8. October 1889 in die Klinik aufgenommen.

Mit 3-4 Jahren traten bei dem bis dahin ganz gesunden Kinde plötzlich täglich 3-5 mal Krämpfe, die der Arzt als Zahnkrämpfe bezeichnete, ein; dies hielt 8 Tage an. Seitdem soll Lähmung der Zunge und des Gesichts bestehen, während die Extremitäten frei blieben. Vor Eintritt der Krämpfe soll Patientin Worte wie "Ja" und "Mama" gesprochen, nachher aber die Sprache ganz verloren haben. Sie spielte aber wie andere Kinder, wusste sich auch verständlich zu machen.

1878 , also im Alter von 3 Jahren, wurde Pat. in der Ambulanz der Klinik gezeigt. Nach den damals gemachten Aufzeichnungen war sie zur Zeit gut entwickelt und konnte gut hören. Starke Speichelabsonderung. Pat. konnte nicht gut schlucken und die Speisen nach hinten in den Mund bringen. Keine Gaumen- und Facialislähmung. Leichte Verschieblichkeit des Unterkiefers. Zunge wölbte sich beim Schreien ganz gut. Fontanelle geschlossen.

Dann wurde Pat. im August 1889 wieder in der Klinik vorgestellt: Starker Speichelfluss, Unfähigkeit, den Mund zu schliessen, die Speisen ohne Mithülfe der Finger in den Schlund zu bringen, - die Zunge herauszustrecken.

Daher wurde der Kranken gerathen, sich aufnehmen zu lassen.

Bei der Aufnahme am 8. October 1889 wurde folgender Status erhoben: Mässig genährtes, für ihr Alter etwas kleines Mädchen. Kopf etwas gross; Umfang $52 \mathrm{Cm}$., nicht difform. Unterkiefer etwas weit hinter 
dem Oberkiefer zurückliegend, untere Schneidezähne $1 \mathrm{Cm}$. hinter den oberen; dieselben können bis in die Nähe der oberen gebracht werden, doch nicht vor dieselben. Mund gewöhnlich etwas offen. Fortwährender Ausfluss von Speichel, dadurch Excoriation der Unterlippe. Bei Nacht hört der Speichelfluss auf.

Gesichtsmusculatur kann nur wenig bewegt werden, am meisten noch die Kinnmusculatur.

Runzeln der Stirn und Augenbrauen nur ziemlich schwach möglich.

Manchmal unwillkürliche Bewegungen der Unterlippe, der Mundwinkel bei Affecten. Willkürlich kann dies nicht geschehen. Lidschluss beiderseits gut.

Keine Störung der Augenmuskelbewegungen.

Pupillen reagiren gut auf Licht und bei Accomodation.

Gehör und Gesicht gut.

Vorderer Theil der Zunge atrophisch, hinterer ziemlich gut entwickelt.

Zunge kann nicht herausgestreckt werden, wird auch beim Kauen nur sehr wenig nach innen und unten bewegt. Beim Würgen wird sie dagegen bis über die Zahnreihe nach vorn gestreckt, ihre Oberfläche in eine hohle Rinne umgewandelt.

Gaumensegel dünn, atrophisch.

Beim Trinken von Flüssigkeit kommt leicht etwas in die Nase. Bei Intonation von, ,a" bleibt das Gaumensegel unbeweglich. Beim Würgen wird es dagegen gehoben.

Kraft der Kaumuskeln gut. Verschiebung des Unterkiefers etwas ausführbar.

Beim Essen muss Pat. die Speisen immer wieder mit den Fingern zwischen die Zahnreihen hineinschieben und verhindern, dass dieselben wieder aus dem Munde herausfallen.

Beim Schlucken von Flüssigkeit und festen Speisen geräth leicht etwas in den Larynx. Um die Speisen schlucken zu können, bringt Pat. sie theils mit den Fingern, theils mit dem Loffel bis hinten in den Schlund hinein.

Bei der laryngoskopischen Untersuchnng zeigt sich die Epiglottis sehr klein und dünn, die Aryknorpel ebenfalls sehr mangelhaft entwickelt. Stimmbänder rosaroth, dünn. Bewegung der Aryknorpel nicht sehr ausgiebig, doch schliessen die Stimmbänder ziemlich gut beim Intoniren.

Pat. kann nur Töne wie , a " und ,e" hervorbringen, mit deutlich nasalem Beiklang, dagegen weder die anderen Vocale, noch irgend einen Consonanten; doch verständigt sie sich sehr gut durch Schreiben und zeigt so, dass sie für ihr Alter und ihren Stand eine sehr gute Intelligenz und Kenntnisse besitzt.

Bei der electrischen Untersuchung der Gesichtsmusculatur stellt sich heraus, dass alle Muskeln auf den faradischen Strom gut reagiren, besonders die Kinnmusculatur und der M. orbicularis oculi. Die Oberlippenmuskeln reagiren etwas schwächer, am schwächsten die MM. zygomatici, lateraler Theil des M. orbicularis oris und die Stirnmusculatur. Vom Facialis aus ist die Reaction der Muskeln schwächer, als bei directer Reizung. Auch auf den galvanischen Strom zeigt sich dasselbe Verhalten.

Zungen- und Gaumenmusculatur reagirt auf beide Stromarten. 
Keine Störung in den Extremitäten. Auch an den inneren Organen nichts Abnormes.

Pat. wird nun mit dem faradischen Strom behandelt, und es werden Sprechübungen angestellt.

Am 17. December klinische Vorstellung. Es wird die Diagnose auf abgelaufene Poliomyelitis der Bulbärkerne gestellt.

Der Zustand hat sich etwas gebessert. Mund wird mehr geschlossen gehalten, so dass gewöhnlich nur die Enden der oberen Schneidezähne sichtbar sind, ist weniger breit; doch keine willkürliche Bewegung der Lippenmusculatur. Wird plötzlich eine Electrode in die Nähe des Gesichts gebracht, so tritt reflectorisch vollkommener Schluss des Mundes ein. Derselbe tritt auch beim Weinen ein, so dass Pat., wenn sie aufgefordert wird, den Mund zu schliessen, sich in weinerliche Stimmung zu versetzen pflegt; doch tritt dabei dann, auch oft, ohne dass Pat. dies hindern kann, stärkeres Auseinanderweichen des Mundes ein. Bei geschlossenem Mund kann Pat. dann ",m" und "ma" aussprechen, nur entweicht dabei immer ein Theil der Luft durch die Nase.

Zunge kann so weit nach vorn gebracht werden, dass die Spitze die untere Zahnreihe überragt.

Durch Anpressen der Zunge an den harten Gaumen und plötzliches Entfernen derselben durch starkes Oeffnen des Mundes kann Pat. einen Schnalzlaut willkürlich hervorbringen.

Gaumensegel hebt sich besser bei Intonation von "a" und „,", besonders stark contrahirt sich der M. azygos uvulae.

Schon seit längerer Zeit kommt beim Trinken auch kein Wasser mehr in die Nase, auch verschluckt sich Pat. nicht mehr.

Oft reichliche Ansammlung von Schleim im Rachen und Kehlkopfeingang.

Pat. kann "a" und „e" ziemlich gat, , $\mathrm{i}^{\text {" }}$ " weniger gut, „0" nur un-

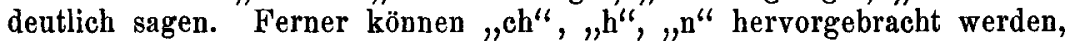
"r" nur undeutlich. Gutturale, dentale und labiale Consonanten können nicht ausgesprochen werden. Combinationen wie "ach", "ne" können oft ziemlich gut ausgeführt werden, aber immer mit grosser Anstrengung.

Der Speichelfluss hat trotz Atropin nicht nachgelassen, der Speichel fliesst besonders stark bei psychischer Erregung und ist gewöhnlich zähschleimig.

Auf dem Lippenroth unterscheidet Pat. nur schlecht Knopf und Spitze der Nadel und localisirt schlecht, während sie auf der angrenzenden Haut scharf unterscheidet.

Sensibilität an der Zunge etwas herabgesetzt, am Gaumen gut.

Die Intelligenz erscheint sehr gut. Pat. schreibt gut Briefe, weiss das Gelesene inhaltlich richtig und in guter Form wiederzugeben; ihr Gedächtniss erscheint sehr gut.

Pat. geht mit etwas nach vorn gebeugter Brustwirbelsäule, so dass sie Gehübungen machen muss. Doch ist eine deutliche Schwäche der Muskeln nicht nachzuweisen.

Appetit immer sehr gut.

Pat. hat um 2,4 Kilo zugenommen. 
Pat. übersteht dann Ende December Influenza ohne wesentliche Störungen.

Im März 1890 Laryngitis, Bronchitis, Angina follicularis.

Am 21. März 1890 wird Pat. entlassen.

Bei der Entlassung ist die Mundspalte im Allgemeinen kleiner als früher, kann aber nicht spontan geschlossen werden.

Immer noch starke Salivation.

Speisen kann Pat. besser im Mund halten, muss nur selten mit dem Finger nachhelfen.

Gaumensegel hebt sich bei Intoniren gut.

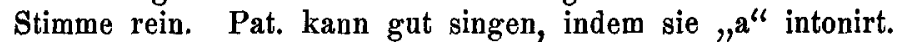

Electrische Untersuchung. Auf den faradischen Strom reagiren :

Kinnmusculatur, M. orbicularis oculi, M. corrugator bei $100 \mathrm{Mm}$. Rollenabstand, MM. zygomatici, orbic. oris, Zunge $=90=$ Gaumensegelmusculatur $\quad=80=$ Vom Facialisstamm aus Reaction $=65=$

Zuckungen etwas träge, beiderseits gleich.

Galvanisch. Kinnmusculatur: KaSZ $1 \frac{1}{2}$ M.-A.

$\begin{array}{ll} & \text { AnSZ } 3= \\ \text { Gröic. oris: } & \text { KaSZ } 2= \\ & \text { AnSZ } 2=\text { schwächer. } \\ \text { Zygomatici: } & \text { KaSZ } 2= \\ & \text { AnSZ } 31 / 2= \\ \text { N. facialis : } & \text { KaSZ 3 }= \\ & \text { AnSZ } 31 / 2= \\ & \text { AnOZ } 8= \\ \text { Zunge: } & \text { KaSZ } 2= \\ & \text { AnSZ 3 }=\end{array}$

Im April 1890 wurde Pat. wieder für 7 Wochen aufgenommen und die faradische Behandlung der Gesichts-, Zungen- und Gaumenmusculatur von Neuem begonnen.

Der Zustand war bei der Aufnahme unverändert und blieb es auch. Die electrische Erregbarkeit der Musculatur erschien normal.

Bei dem geschilderten Falle bestand eine Lähmung im Gebiete des Facialis, des Hypoglossus und Accessorius, die betroffenen Muskelgruppen zeigten im Beginn der Beobachtung theilweise herabgesetzte Erregbarkeit, sie waren atrophisch, die Reflexe waren aber erhalten.

Gegen eine periphere Erkrankung spricht der Beginn der Krankheit mit Krämpfen u. s. w., also Erscheinungen von Seiten des Centralnervensystems, ferner die gleichmässige symmetrische Ausbreitung, das Fehlen stärkerer Entartungsreaction.

Man könnte ferner an einen Process jim Grosshirn denken, analog den Fällen von sogenannter Pseudobulbärparalyse, wo von zwei 
symmetrischen Herden im Centrum derselben, oder auch nur von einem ausgehend, eine Lähmung der von diesen Nerven versorgten Muskelgruppen zu Stande kam. Aber wenn man die in letzter Zeit in der Arbeit von Leresche ${ }^{1}$ ) sorgfältig zusammengestellten Fälle dieser Art genauer betrachtet, so handelt es sich dabei immer auch um erhebliche Störung der Intelligenz, es handelt sich meist um alte Leute, um Apoplexien, Embolien im Gehirn. Die Muskeln atrophiren nicht dabei.

Hier aber ist Muskelatrophie vorbanden und dabei eine sehr gute Intelligenz.

Am besten lässt sich das ganze Krankheitsbild wobl erklären, wenn man eine Degeneration der Nervenkerne in der Medulla oblongata annimmt.

Von der dort sich abspielenden progressiven Bulbärparalyse unterscheidet sich der Fall dadurch, dass er stationär geblieben ist; auch handelt es sich bei dieser Affection fast ausschliesslich um ältere Leute, wenn auch Fälle, wie der von Hoffmann ${ }^{2}$ ) jüngst beschriebene, zeigen; dass sie auch im Kindesalter in typischer Form vorkommen kann. Ferner entwickelt sich diese Krankheit schleichend, während hier ein acuter Beginn vorhanden ist.

Der acute Anfang mit Krämpfen im ersten Lebensjahr, an die sich die Lähmung unmittelbar anschloss, hat die grösste Aehnlichkeit mit dem Auftreten der acuten Kinderlähmung. Daher erseheint die Annahme noch am wahrscheinlichsten, dass es sich in unserem Fall zunächst um acute Entzündung der Kerne des Facialis, Hypoglossus und Accessorius handelte, eine Bulbärmyelitis, bei der die vitalen Centren nicht betheiligt waren, und die eine abnorme Localisation der Poliomyelitis anterior acuta der Kinder darstellte. Wie bei dieser Erkrankung, blieben Defecte in den Kernen zurïck, die zum Ausfall der von ihnen abhängigen Muskelgruppen führten. Dabei sind die intramedullären Bahnen der betreffenden Nerven wohl intact geblieben, da die Reflexe noch vorhanden sind, analog dem Fall von acuter Bulbärmyelitis von $\mathrm{Etter}^{3}$ ), wo die Reflexe trotz der Lähmung auf der rechten Seite erhalten waren, wo die Erkrankung nur die Kerne betraf, dagegen auf der anderen Seite feblten, weil dort der Herd die Bahnen der Nerven unterbrach. Dass bei der ersten Untersuchung im 4. Lebensjahr bei unserer Kranken keine Facialis- und Gaumensegellähmung gefunden wurde, beruht wohl

1) Etude sur la Paralysie glosso-labiée cérébrale. Paris 1890.

2) Deutsche Zeitschr. f. Nervenheilkunde. Bd. I. S. 169.

3) Corresp.-Blatt f. Schweizer Aerzte. 1882. Nr. 24. 
darauf, dass auf sensible Reize die betreffenden Muskeln reagirt haben werden, willkürliche Bewegungen dieser Organe bei dem Kinde aber wobl nicht erzeugt werden konnten. Die Angabe der Eltern steht damit auch im Widerspruch, indem diese die Lähmung des Gesichts schon gleich nach Beginn gefunden haben und ferner das Sprechen auch schon gleich unmöglich wurde.

In der Literatur finden sich nur wenige Angaben über das Vorkommen einer acuten Poliomyelitis der Medulla oblongata. So erklärt Eisenlohr einen Fall von acut eintretender gekreuzter Hemiplegie mit zurückbleibender geringer linksseitiger Facialislähmung in dieser Weise. Bei der Section fand sich auch einige Jahre später eine degenerative Atrophie des linken Facialiskerns und -stammes und der zugehörigen Muskeln, nichts im Riückenmark.

Auch sind von Medin ${ }^{1}$ ) bei einer in Stockholm herrschenden Epidemie von infantiler Paralyse 3 Fälle von Monoplegia facialis, Störungen des Hypoglossus, Lähmung des Accessorius, Vagus, Oculomotorius beobachtet worden.

Es fanden sich zum Theil entzündliche degenerative Veränderungen in den Kernen des Hypoglossus, Vagus, Facialis and Abducens bei den zur Section gekommenen Fällen.

Auch die Fälle von angeborener Bulbärlähmung sind wohl auf solche Vorgänge in Kernen der Medulla zum Theil zurïckzuflihren.

So beschreibt Hitzig ${ }^{2}$ ) den Fall eines 6 jährigen Mädchens mit Lähmung von Gesichtsmuskeln, Zunge, Gaumensegel, Speichelfluss, Parese des rechten Rectus internus, Unvermögen, andere Laute, als Vocale und Hauchlaute hervorzubringen. Hier trat unter galvanischer Behandlung und Uebung fast vollkommene Heilung ein. Hitzig nimmt eine angeborene Affection der Nervenkerne am Boden der Rautengrube an.

Von Berger ${ }^{3}$ ) sind ferner Fälle beschrieben worden von Parese oder Paralyse der an der Articulation und Deglutition betheiligten Bulbärnerven, Salivation; manchmal waren dabei Hemiparese und verschiedene Missbildungen vorhanden. Auch er führt dies auf intrauterine Erkrankungen oder Entwicklungshemmung zurück.

Ein derartiger Fall von angeborener Lähmung bulbärer Nerven kam in diesem Jahre auch in der Klinik vor und möchte ich diesen hier noch kurz anfügen.

1) En epidemi af infantil paralysi. Hygiea LII. 9. p. 657. Referat: Schmidt's Jahrbücher 189.1. S. 245.

2) Berl. klin. Wochenschr. 1874. S. 465.

3) Nach Eulenburg's Realencyklopädie. Bd. III. S. 580. Das Original konnte ich nicht auffinden. 
Bett y Frenzen, 5 Jahre alt, kann seit der Geburt das Gesicht nicht bewegen. Geburt soll etwas erschwert gewesen sein, doch wurde keine ärztliche Hülfe, speciell nicht die Zange angewandt. Bei Schreien und Lachen wurde der Mund nie verzogen, doch sollen beim Lachen manchmal Grubchen in den Wangen aufgetreten sein, was sich später verlor. Die Augenlider konnten nie ganz geschlossen werden, der Speichel lief häufig aus dem Munde.

Im Alter von 2 Jahren wurde die Störung des Gehvermögens mit stärkerer Betheiligung des rechten Beines zuerst bemerkt, nachdem schon seit Ende des 1. Jahres Gehversuche gemacht worden waren.

Mit $3 \frac{1}{2}$ Jahren Masern, danach stärkere Gehstörung.

Vor einem Jahr sollen einmal für einige Minuten Krämpfe und Bewusstlosigkeit eingetreten sein. Sonst hat Patientin nie derartige Störungen gehabt.

Am 28. Mai 1891 wurde Pat. in die Klinik aufgenommen.

Bei dem mässig genährten Kinde fällt sofort die fast vollkommene $\mathbf{A}$ b wesenheit der mimischen Bewegungen im Gesicht auf; weder beim Weinen, noch beim Lachen zeigt das Gesicht eine wesentliche Veränderung, nur die Unterlippe wird dabei etwas mimisch bewegt. Fast keine Faltenbildung im Gesicht.

Der Mund steht häufig offen, kann geschlcasen werden, doch wird dabei die kurze dünne Oberlippe nicht bewegt, nur die Unterlippe nach oben geschoben. Der Mund kann nicht zum Pfeifen gespitzt werden, während Blasen gut erfolgt.

Im Schlaf steht der Mund offen, wird aber meist durch die vorgeschobene Zunge versehlossen, so dass Pat. dann nur durch die Nase athmet.

Die Wangen sind schlaff, doch enthalten sie anscheinend reichlich Fett.

Von den Nasenmuskeln wird nur der M. levator alae nasi proprius beim Weinen, tiefen Inspiriren bewegt.

A ugen lider können nicht vollkommen geschlossen werden. Oberer Augenlidknorpel sehr dünn. Hebung erfolgt gut, Action des M. orbicularis ganz fehlend.

Stirn kann nicht gerunzelt werden. Sensibilität im Gesicht anscheinend normal.

Bewegungen der A agen gut.

Z unge etwas schmal, wird gerade und ohne Zittern heransgestreckt.

Ga umensegel etwas dünn, wird nur mit geringer Energie gehoben.

Pat. kann gut schlucken, verliert beim Kauen nichts aus dem Munde. Kaumuskeln gut functionirend.

Pat. kann gut sprechen, auch die Lippenbuchstaben werden gut ausgesprochen, wenn auch mit geringer Energie, indem Pat. dieselben durch Hinaufschieben und Anpressen der gut beweglichen Unterlippe an die unbewegliche Oberlippe hervorbringt. " $R^{\prime}$ und die Zischlaute machen ihr Schwierigkeiten. Die Sprache ist etwas näselnd, infolge mangelhaften Abschlusses gegen die Nase.

Stimme rein.

Hören und Sehen gut.

Intelligenz für das Alter der Pat. sehr gut entwickelt.

Arme etwas wenig entwickelt, besonders erscheint der Biceps beider- 
seits ziemlich gering entwickelt, doch können alle Bewegungen gut ausgeftuhrt werden.

Grobe Kraft, besonders bei Extension, gut. Händedruck kräftig.

Sensibilität nicht gestört.

Aufrichten aus liegender Stellung ohne Unterstützung der Arme nicht möglich.

Ba uchmuskeln in den unteren Portionen ziemlich schwach, wölben sich beim Husten, besonders rechts, vor.

Musculatur der Beine etwas dïnn und schlaff.

Gang schwankend; Pat. überkreuzt dabei die Beine, linkes Bein wird dabei stark nach aussen gedreht. Dabei ziemlich starke Lordose der Lendenwirbelsäule, das Becken wird stark mitbewegt.

Stehen auf dem linken Bein schlechter als auf dem rechten.

Linke Oberschenkelmusculatur, besonders in den Adductoren, schwächer als die rechte, ebenso die Glutäalmusculatur besonders links schlaff. Linker Tibialis anticus dünner als der rechte.

Schmerzempfindung vielleicht in den Beinen herabgesetzt, Tastempfindung gut.

Patellarsehnenreflex undentlich. Achillessehnenreflex, Fusssohlenreflex gut. normes.

Blase und Mastdarm nicht gestört. Innere Organe zeigen nichts $\mathrm{Ab}$ -

Electrische Untersuchung. Gesicht: Vom N. facialis aus reagiren auf den faradischen Strom, beiderseits ungefähr gleich, nur die Kinnmusculatur und der Levator alae nasi.

Auch direct sind nur diese Muskeln erregbar.

Galvan is ch: Facialis KaSZ und AnSZ beiderseits $3 \frac{1}{2}$ M.-A., gleich stark. Es zucken die oben genannten Muskeln, dieselben reagiren auch allein auf den galvanischen Strom direct.

Untersuchung durch Weinen sehr erschwert.

$\mathrm{B}$ e in e: M. tibialis anticus. Faradisch :

links 100 R.-A.

rechts $90=$

Galvanisch: links KSZ 3 M.A.

rechts $=2 \frac{1}{2}=$

AnSZ - KaSZ.

Von N. peroneus aus:
Faradis ch: links 105 R.-A. rechts $100=$
Galvanisch beiderseits: KaSZ 1 M.-A.
$\operatorname{AnOZ} 1 \frac{1}{2}=$
AnSZ 2 =

M. gastrocnemius und soleus:

Faradisch links und rechts $110 \mathrm{R}$.-A., ebenso vom Nerven aus.

Bei directer faradischer Reizung des Tibialis überwiegt die zugleich auftretende Zuckung der Wadenmusculatur.

Oberschenkel:

M. quadriceps, faradisch: links 67 R.-A. 
196 IX. HoPFE-SEYLER, Erkrankung der Medulla oblongata im Kindesalter.

$$
\begin{aligned}
& \text { Galvanisch: links KaSZ } 4 \text { M.-A. } \\
& \text { AnSZ } 7= \\
& \text { rechts KaSZ } 4 \text { = } \\
& \text { AnSZ } 6=
\end{aligned}
$$

Adductoren. Faradisch: links 50 R.-A. rechts $54=$

M. tensor fasciae latae beiderseits. Faradisch: 67 R.-A.

Galvanisch: KaSZ 9 M.-A.

M. glutaei. Faradisch beiderseits; 66 R.-A.

Galvanisch: KSZ und AnSZ 10 M.-A.

$$
\mathrm{KSZ}>\mathrm{AnSZ} \text {. }
$$

Patientin wurde bis zum 24. Juli 1891 mit dem faradischen Strom behandelt, ferner musste sie Gehübungen machen. So wurde ein sichererer Gang und eine bessere Haltung erzielt. Die Störungen im Gesicht blieben unverändert.

Die später erst auftretende, durch die Masern begünstigte Lähmung der Bein- und Beckenmusculatur, die mit Atrophie, Störungen der electrischen Erregbarkeit einherging, ist wohl zu beziehen auf eine Erkrankung der Vorderhörner, auf eine Poliomyelitis anterior lumbalis, die jetzt zum Stillstand gekommen ist.

Dagegen beruht wohl die Lähmung des Gesichts auf einer intrauterinen Erkrankung eines Theils der Kerne des Facialis und Zugrundegehen oder Entwicklungshemmung des grössten Theils seiner Fasern und der von ihm sonst versorgten Muskeln. ${ }^{1}$ )

1) Vgl. die soeben erschienene Arbeit von $M$ öbius: Ueber infantilen Kernschwund. Münchener med. Wochenschrift 1892. Nr. 2-4. 